\title{
Brain MRI Segmentation and Bias Estimation Via An Improved Non-Local Fuzzy Method
}

\author{
Yunjie Chen ${ }^{1}$, Zhengkai Wang ${ }^{1}$, Jin Wang ${ }^{2}$ and Yuhui Zheng ${ }^{2}$ \\ ${ }^{1}$ School of math and statistics, Nanjing University of Information \\ Science \& Technology, Nanjing 210044, China \\ ${ }^{2}$ School of Computer \& Software, Nanjing University of Information \\ Science \& Technology, Nanjing 210044, China
}

\begin{abstract}
Intensity in homogeneities cause considerable difficulties in the quantitative analysis of Magnetic Resonance (MR) images. Thus intensity in homogeneities estimation is a necessary step before quantitative analysis of MR data can be undertaken. This paper proposes a new energy minimization framework for simultaneous estimation of the intensity in homogeneities and segmentation. The intensity in homogeneities is modeled as a linear combination of a set of basis functions, and parameterized by the coefficients of the basis functions. The energy function depends on the coefficients of the basis functions, the membership ratios and the centroids of the tissues in the image. Intensity in homogeneities estimation and image segmentation are simultaneously achieved by calculating the result of minimizing this energy. Furthermore, in order to improve its robustness to noise, the membership ratios are adapted by using nonlocal information. Experimental results on both real MR images and simulated $M R$ data show that our method can obtain more accurate results when segmenting images with bias field and noise.
\end{abstract}

Keywords: Non-local Information, Basis Function, Bias field, FCM, Noise

\section{Introduction}

Magnetic resonance imaging (MRI) is a helpful method for diagnoses of brain illness. In MRI data analysis, precise segmentation of different tissues plays a pivotal role for brain studies. Although many algorithms have been reported, automated segmentation still remains a difficult task due to the intensity corruption caused by noise and intensity in homogeneity. Intensity in homogeneity, which is also named bias field, usually makes the intensity of the same tissue varies with the location of the tissue. Therefore, correction for such intensity in homogeneities is often a mandatory step before quantitative analysis of the image data.

There are many methods have been reported to correct or estimate the intensity in homogeneities. [1-2]. Some correction methods rely on measuring the coil sensitivity functions [3]. These approaches can correct for coil sensitivities but cannot correct for other sources of intensity in homogeneities. In other words, intensity in homogeneities will vary with different machines and acquisition parameters from subject to subject and from slice to slice. All these drawbacks challenge the validity of these methods. In contrast to these coil sensitivity functions based methods, some researcher's estimated bias field by using the information within the acquired image [13].

Recently, an increasing number of people utilize segmentation based approaches to estimate intensity in homogeneities [4, 13]. Intensity in homogeneities estimation is a necessary preprocessing step enabling better image segmentation. On the other hand, correct segmentation makes bias field correction rather trivial. Bias field correction and segmentation can thus be viewed upon as two intertwined procedures. In segmentation based methods these 
two procedures are merged so that they benefit from each other, simultaneously yielding better segmentation and bias field correction.

The maximum-likelihood or the maximum a posteriori probability criterion is one of the most popular segmentation methods. Wells et.al [4] extended the framework of maximum likelihood classification to estimate the bias field. Finite Gaussian mixture model is used and modified to incorporate bias fields. The parameters of the model can be estimated by using the expectation-maximization (EM) algorithm.

The model can yield impressive results on brain MR images but has the disadvantage of being computationally intensive and requiring initialization of explicitly modeled classes and spatial distribution of tissues. The initialization usually be obtained by manual selection of representative points for each class, however, such initializations are subjective and often irreproducible. So as to overcome this shortcoming, Andersen et al. [5] proposed an automatic approach to initialize the parameters by registering set of images.

As we known that the intensity probability models do not take account of any spatial dependency among image pixels belonging to the same class, which makes the models sensitive to noise. In order to reduce the effect of the noise, Markov random fields have been incorporated [6] which is less sensitive to noise and can obtain smoother borders. However, to avoid over smoothing of tissue borders, spatial connectedness should not be too strong.

Some methods introduce the fuzzy c-means (FCM) method and modify the objective function to segment images meanwhile estimating the bias field. FCM is a commonly used clustering approach. Two extended versions of the FCM method have been proposed to estimate the bias field with an additive term in its object function. Pham [7] used first and second-order regularization terms to ensure the smoothness and continuity of the estimated bias field. However, it is hard to find the exact coefficient of the smoothing term to obtain accurate results. Furthermore, this method is computationally intensive, due to the introduction of the bias field smoothing term. Ahmed et.al.[8] incorporated spatial information by adding a spatial regularization term that enabled the class membership of a pixel to be influenced by its neighbors. This approach proved tolerant to salt and pepper noise, resulting in smoother segmentation. However, a regularization parameter also needed to determine the smoothness of segmentation and the bias field.

Li et.al [14] proposed an improved level set method by using a weighted K-means clustering objective function for image intensities in a neighborhood around each pixel. The method can segment images meanwhile estimate the bias file. But this method only bases on the local mean information, which makes the method sensitive to the variance. In addition, this method is also computationally intensive, due to the use of the Level Set. In order to estimate bias field more efficiently, the same authors modeled the bias field as a linear combination of smooth basis functions, and hence parameterized as the coefficients of the basis functions. The bias field can be obtained by minimizing the energy function. A salient advantage of the method is its independence of initialization; however, it cannot obtain more accurate results due to the weakness of the K-means. Besides, this method also sensitive to the noise, in this paper, we propose a new energy minimization approach for simultaneous tissue classification and bias field estimation of MR images. We also modeled the bias field as a linear combination of smooth basis functions, and then parameterized it as the coefficients of the basis functions. The energy function depends on the patch information of every pixel, the coefficients of the basis functions, the membership functions and the centroids of the tissues in the image. Bias field estimation and image segmentation are simultaneously achieved as the result of minimizing this energy. 


\section{Methods}

\subsection{Background}

The observed MRI signal $\mathrm{J}$ is the product of the true signal I generated by the underlying anatomy and spatially varying field factor $\mathrm{B}$, and an additive noise $\mathrm{n}$ :

$$
J_{i}=\left(I_{i}+n_{i}\right) \cdot B_{i}, \quad \forall i \in\{1, \ldots, N\}
$$

where, $I_{i}, J_{i}$ are the true and observed intensity at the ith pixel, respectively; $B i$ and $n i$ are the bias field and additive noise at the ith pixel, respectively; $N$ is the total number of pixels in the MRI. Many methods to estimate bias field and segment tissues in MRI are based on three assumptions: (1) Bias field $B$ is smooth and slowly varying. (2) Within the true signal $I$, all pixels of the same tissue have similar true intensities. (3) The true signal $I$ has $K$ tissues in the region of interest. In brain MRI, $K=3$ for white matter (WM), gray matter(GM) and cerebrospinal fluid(CSF).

\subsection{Objective Function}

The standard FCM objective function for partitioning I into K clusters:

$$
E=\int_{\Omega} \sum_{k=1}^{K} u_{k}^{q}(x)\left\|I(x)-v_{k}\right\|^{2} d x
$$

Where $\Omega$ is the brain tissue region, $v_{k}$ is the centroid of class $k$ and $u_{k}(x)$ is the membership value at pixel $x$ for class $k$ such that $\sum_{k=1}^{k} u_{k}(x)=1$. The total number of class $K$ is assumed to be known. The parameter $q$ is a weighting exponent on each fuzzy membership and determines the amount of "fuzziness" of the resulting classification. If $q=1$, then the algorithm degenerates to K-means clustering algorithm. The operator $\|\cdot\|$ is any inner product norm on $R^{M}$, where $M$ is the number of channels or spectra in the image and $\|\cdot\|=\sqrt{\langle\cdot, \cdot\rangle}$.

The FCM objective function is minimized when high membership values are assigned to voxels whose intensities are close to the centroid of its particular class, and low membership values are assigned when the voxel data is far from the centroid. Taking the first derivatives of Eq. 2 with respect to $u$ and $v$ and setting those equations to zero yield necessary conditions for Eq. 2 to be minimized. Iterating through these two necessary conditions leads to a grouped coordinate descent scheme for minimizing the objective function. The advantages of FCM are that it is unsupervised and robust to the initialization in most conditions. However, FCM assumes that the centroids of the image are spatially invariant, which is not true of images that have been corrupted by the bias field.

We proposed a new objective function, which preserves the advantages of FCM while taking the bias field into account. In this paper, we only propose the function for $2 \mathrm{D}$ images, because it is similar for 3D data. The function is written as:

$$
E=\int_{\Omega} \sum_{k=1}^{K} \int_{\Omega} W(x, y) u_{k}^{q}(y) d y\left\|J(x)-B(x) v_{k}\right\|^{2} d x
$$




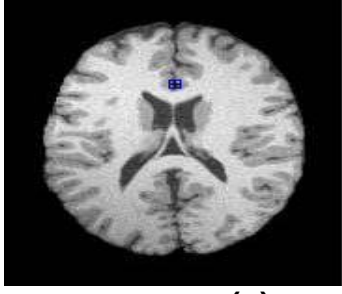

(a)

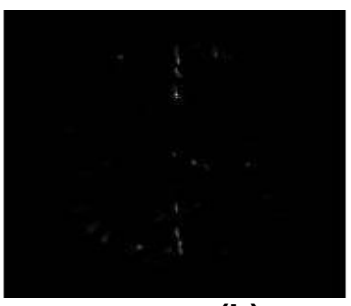

(b)

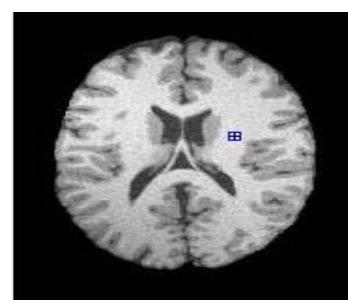

(c)

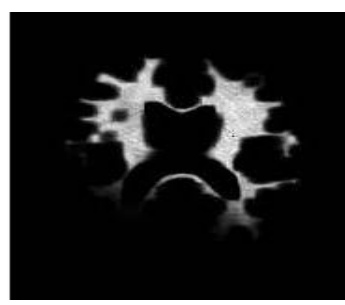

(d)

Figure 1. Description of the Similarity between a Voxel to Others (A) Initial Image (B) Similarity (C) Initial Image (D) Similarity

where $\left.u_{k}(x) \in\left\{[0,1] \mid \sum{ }_{k=1}^{K} u_{k}(x)=1, \forall x\right\}, \forall \mathrm{x}\right\} . W(x, y)$ is a weight function, which depends on the similarity between the neighborhood of $x$ and $y$, to reduce the effect of the noise. The similarity between $\mathrm{x}$ and $\mathrm{y}$ depends on the neighborhood $\mathrm{x}$ and $\mathrm{y} . \mathrm{x}$ is a fixed size square window with width $2 \times p+1$ centered at $\mathrm{x}$. $\mathrm{y}$ is centered at $\mathrm{y}$ with the same width as $\mathrm{x}$. The point $\mathrm{y}$ with a more analogous neighborhood will have a larger weight. Broadly speaking, if the neighborhoods of two pixels $\mathrm{x}$ and $\mathrm{y}$ are similar, it is more probable that these pixels belong to the same tissue and so the weight function increases. Conversely, if these two pixels are quite different in the original image, the influence of the weight function should be decreased, since there is a lower probability that the pixel y might have a strong influence on the classification of the current pixel $\mathrm{x}$. The weight function is defined as:

$$
W(x, y)=e^{-\frac{\|\Delta x-\Delta y\|_{2}^{2}}{h^{2}}}
$$

where $h$ acts as a filtering parameter to control the decay of the exponential function, $\|\Delta x-\Delta y\|_{2}^{2}$ is the Euclidean distance, broadly speaking a small neighborhood around a pixel may match neighborhoods around other pixels within the same image. In practical implementation, the spatial neighborhood is often restricted in a small search window with radius $r$ for computational purpose, instead of the entire image domain $\Omega$.

Figure 1 is a brain MR image from Brain Web brain with the parameter noise level $3 \%$ and intensity in homogeneity level $80 \%$ (mcgill, http:// www. bic. mni. Mcgill. ca/brain web/). Figure 1.a shows the initial image. A pixel $\mathrm{x}$, belongs to CSF, is marked as a black plus, whose neighborhood $\Delta x$ is marked as a blue box with size $7 \times 7$. Figure $1 . b$ indicates the similarities of other pixels' neighborhoods to $\Delta x$. Figure 1.c is the same initial image and a different pixel, belongs to WM, and is located in a different place. Figure 1.d is the similarities of the pixel in Figure 1.c. It can be seen that the pixels, which have more similar neighborhoods, have higher weights. With the non-local region information, the effect of the noise can be reduced easily.

Because the bias field $B$ is usually very smooth across the whole image, we assume that $B$ belongs to a family of smooth functions. We have chosen $B$ to be a linear combination of $L$ smooth basis functions $s_{1}, s_{2}, \ldots, s_{L}$, , which has been shown in [13]:

$$
B(x)=\sum_{l=1}^{L} w_{l} s_{l}(x)
$$

where $w_{l} \in R, l=1, \ldots, L$, are the combination coefficients. Orthogonal polynomials are usually used as the basis functions, which satisfy

$$
\int_{\Omega} s_{i}(x) s_{j}(x) d x=\delta_{i, j}
$$

where $\delta_{i, j}=1$ for $i=j$ and $\delta_{i, j}=0$ for $i \neq j$.

In this paper, we use Legendre polynomials as the basis functions. In 2-D, we selected products of Legendre polynomials $P$ in $x$ and $y$ as the basis functions si. In 3-D, Legendre 
polynomials in $x, y$ and $z$ are used. The image coordinates are scaled to the range $[-1,1]$. For Legendre polynomials up to the degree $m$, the size $L$ of the parameter vector is given for the 2-D case by $L=(m+1)(m+2) / 2$ and for the 3 -D case by $L=(m+1)(m+2)(m+3) / 6$. The choice of the maximal degree of Legendre polynomials largely hinges on prior knowledge of the coil and the expected type and smoothness of the bias field.

The parameters $w_{1}, w_{2}, \ldots, w_{L}$ and $s_{1}, s_{2}, \ldots, s_{L}$ can be represented in the form of column vectors, i.e. $w=\left(w_{1}, w_{2}, \ldots, w_{L}\right)^{T}$ and $S(x)=\left(s_{1}(x), s_{2}(x), \ldots, s_{L}(x)\right)^{T}$. Consequently, the energy function above can be updated as:

$$
E=\int_{\Omega} \sum_{k=1}^{K} \int_{\Omega} W(x, y) u_{k}^{q}(y) d y\left\|J(x)-w^{T} S(x) v_{k}\right\|^{2} d x
$$

Minimization with respect to $u$. Eq.7 will be written with its constrained condition by using one Lagrange multiplier:

$$
E=\int_{\Omega} \sum_{k=1}^{K} \int_{\Omega} W(x, y) u_{k}^{q}(y) d y\left\|J(x)-w^{T} S(x) v_{k}\right\|^{2} d x+\lambda\left(1-\sum_{k=1}^{K} u_{k}(x)\right)
$$

It also can be written as

$$
\begin{aligned}
E & =\int_{\Omega} \int_{\Omega} \sum_{k=1}^{K} \int_{\Omega} W(x, y) u_{k}^{q}(y) d y\left\|J(x)-w^{T} S(x) v_{k}\right\|^{2} d x d y+\lambda\left(1-\sum_{k=1}^{K} u_{k}(x)\right) \\
& =\int_{\Omega} \sum_{k=1}^{K} u_{k}^{q}(x) \int_{\Omega} W(x, y)\left\|J(x)-w^{T} S(x) v_{k}\right\|^{2} d y d x+\lambda\left(1-\sum_{k=1}^{K} u_{k}(x)\right)
\end{aligned}
$$

Taking the derivative of $E$ with respect to $u_{k}(x)$ and setting the result to zero, we have, for $p>1$

$$
\left.\left[\frac{\partial E}{\partial u_{k}(x)}=q u_{k}^{q-1}(x) \int_{\Omega} W(x, y)\left(J(x)-w^{T} S(x) v_{k}\right)^{2} d y-\lambda\right]\right|_{u_{k}(x)=u_{k}^{*}(x)}=0
$$

Solving for $u_{k}^{\#}(x)$ we have

$$
u_{k}^{\#}(x)=\left(\frac{\lambda}{q \int_{\Omega} W(x, y)\left(J(x)-w^{T} S(x) v_{k}\right)^{2} d y}\right)^{\frac{1}{q-1}}
$$

Since $\sum_{k=1}^{K} u_{k}(x)=1, \forall x$, we have

$$
\sum_{k=1}^{K}\left(\frac{\lambda}{q \int_{\Omega} W(x, y)\left(J(x)-w^{T} S(x) v_{k}\right)^{2} d y}\right)^{\frac{1}{q-1}}=1
$$

Then

$$
\lambda=\frac{q}{\left(\sum_{k=1}^{K}\left(\frac{1}{\int_{\Omega} W(x, y)\left(J(x)-w^{T} S(x) v_{k}\right)^{2} d y}\right)^{\left.\frac{1}{q-1}\right)^{q-1}}\right.}
$$

Substituting into Eq.10, the zero-gradient condition for the membership estimator can be rewritten as

$$
u_{k}^{\#}(x)=\frac{\frac{1}{\left(\int_{\Omega} W(x, y)\left(J(x)-w^{T} S(x) v_{k}\right)^{2} d y\right)^{\frac{1}{q-1}}}}{\sum_{k=1}^{K} \frac{1}{\left(\int_{\Omega} W(x, y)\left(J(x)-w^{T} S(x) v_{k}\right)^{2} d y\right)^{\frac{1}{q-1}}}}
$$


Minimization with respect to $v$ for fixed $u$ and $W$, taking the derivative of $E$ with respect to $v_{k}$ and setting the result to zero, we have

$$
\left.\left[\frac{\partial E}{\partial u_{k}(x)}=2 \int_{\Omega} \int_{\Omega} W(x, y) u_{k}^{q}(y)\left(J(x)-w^{T} S(x) v_{k}\right) d x d y\right]\right|_{v_{k}(x)=v_{k}^{*}}=0
$$

Solving for $v_{k}^{\#}$ we have

$$
v_{k}^{\#}=\left(\frac{\int_{\Omega} \int_{\Omega} W(x, y) u_{k}^{q}(y) J(x) d x d y}{\int_{\Omega} \int_{\Omega} W(x, y) u_{k}^{q}(y) w^{T} S(x) d x d y}\right)
$$

Minimization with respect to $w$ for fixed $u$ and $v$, taking the derivative of $E$ with respect to $w$ and setting the result to zero, we have

$$
\begin{aligned}
& {\left[\frac{\partial E}{\partial W}=-2 \int_{\Omega} \int_{\Omega} \sum_{k=1}^{K} W(x, y) u_{k}^{q}(y)\left(J(x)-w^{T} S(x) v_{k}\right) S(x) d x d y\right]} \\
& =-2 \int_{\Omega} \int_{\Omega} \sum_{k=1}^{K} W(x, y) u_{k}^{q}(y)(J(x) S(x) d x d y \\
& \quad+-2 \int_{\Omega} \int_{\Omega} \sum_{k=1}^{K} W(x, y) u_{k}^{q}(y) w^{T} S(x) S(x) d x d y \\
& =-2 \int_{\Omega} \int_{\Omega} \sum_{k=1}^{K} W(x, y) u_{k}^{q}(y) J(x) S(x) d x d y \\
& \left.\quad+-2 \int_{\Omega} \int_{\Omega} \sum_{k=1}^{K} W(x, y) u_{k}^{q}(y) S(x) S(x)^{T} d x d y \quad w\right]_{w=w^{*}}=0
\end{aligned}
$$

Note that $A=\int_{\Omega} \int_{\Omega} \sum_{k=1}^{K} W(x, y) u_{k}^{q}(y) S(x) S(x)^{T} d x d y$ is an $L \times L$ matrix, where $L$ is the number of the basic functions $G=\int_{\Omega} \int_{\Omega} \sum_{k=1}^{K} W(x, y) u_{k}^{q}(y) J(x) S(x) d x d y$ is a vector of the same size as w. Solving for $w^{\#}$, we have

$$
w^{\#}=A^{-1} G
$$

The entire procedure correcting the bias field and segmenting the image into different clusters can be summarized in the following steps.

Step 1. Initialize $\mathrm{v}, \mathrm{u}$ and $\mathrm{w}$;

Step 2. Update $u$ to $u^{\#}$ by Eq. 13;

Step 3. Update $w$ to $w^{\#}$ by Eq. 17;

Step 4. Update $v$ to $v^{\#}$ by Eq. 15;

Step 5. Check convergence criterion. If convergence has been reached, stop the iteration, otherwise, go to Step 2.

\section{Implementation and Results}

Our method was applied in MATLAB on a PC with Intel Pentium IV 2.4G processor and 2048 RAM. In order to test the reliability and validity of the algorithm, we describe the segmentation on synthetic images and real brain MR images. We take the parameters $K=3$, $m=3, p=1, r=6$ and $h=28$ for all the images in this paper. 


\subsection{Evaluation with Simulated Data}

Figure2 shows the segmentation results of a simulated MR image, which was created with the MRI simulator (Brain Imaging Center at the Montreal Neurological Institute, McGill University, mcgill). There are many advantages for using these synthetic images rather than real image data for validating segmentation methods. These advantages include prior knowledge of the true tissue types and control over image parameters such as mean intensity values, noise, and bias field.

We compared our method with FCM and other six segmentation based methods: Wells' method [4], Leemput method [9], AFCM method [8], Li method [13], hidden-MRF-based method (FSL)[6] and NLFCM method[15]. Figure2shows the segmentation results of the 87th transaxial image with the noise levels $5 \%$ and intensity in homogeneity level $80 \%$. Figure2 (a) is the initial image. Figure2 (b) is the ground truth. Figure 2(c) is the segmentation result of FCM method. It can be seen that, due to the effect of the bias field, some of the WM tissues have be misclassified into GM. Figure 2(d-f) are the segmentation results of Leemput's method, Wells' method and Li's method. These three techniques can reduce the effect of the bias field; however, they only use the intensity distribution information, which makes these three methodologies sensitive to the noise. The segmentation result of the AFCM method is exhibited in Figure 2(g). This method extends the FCM method to reduce the effect of the bias field and uses the neighbor information to reduce the effect of the noise. However, it is an isotropic method, which makes it unable to reduce the effect of strong noise. Apart from it, when the size of the neighbor increases, the method will lose structure information of brain tissues easily [33]. Figure 2(h) reveals the result of the hidden-MRF-based method, which reduces the effect of the noise by imposing certain spatial constraints on the classifications. However, this method uses mean field theory to make the method more efficiently, but still sensitive to noise [34].

Figure2 (i-j) are the segmentation results of the NLFCM method and our method, respectively. These two methods are all based on patch information. In order to reduce the effect of the intensity in homogeneity, the NLFCM method defines a specific value, which is computed for each voxel over a local neighborhood, for each point of the image. This method can only reduce the effect of the intensity in homogeneity and cannot obtain bias field. Moreover, this method has high computational cost, due to computing local mean for each point. Our method parameterizes the bias field as coefficients of the basis function, which has less computational complexity and our energy function is convex in each of its variables and converges into the minimization easily. To weaken the effect of the noise, the NLFCM method defines a patch information based regularization term, which extends the idea of the AFCM method. Compared to the NLFCM method, our method has not any regularization term, which leads our method more efficiently with less parameter. From Eq.13, we can find that is based on the patch information. Broadly speaking, the neighborhoods of pixels belong to the same tissue will have higher weight $\mathrm{W}$ and have higher probability that have a strong influence on the classification of current pixel. Because our method can segment images meanwhile estimate the bias field, which makes our method obtain more precise result than that of the NLFCM method.

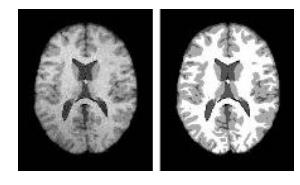

(a) (b) 


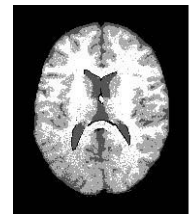

(c)

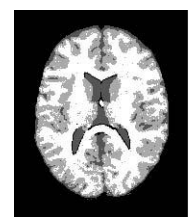

(d)
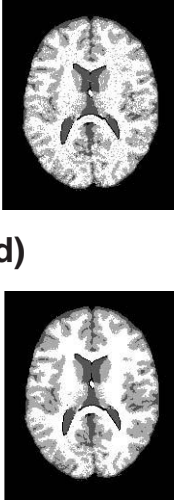

(h)

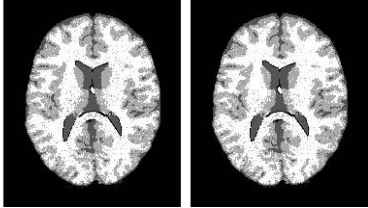

(e)

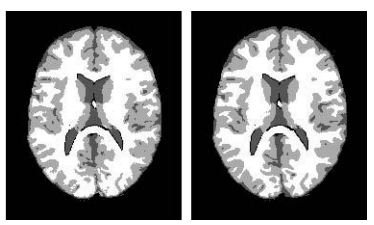

(i) (f)

(j)

Figure 2. Segmentation Results on the 87th Transaxial Image of a Simulated Image Data Set with the Parameter: Noise Levels $5 \%$ and Intensity In Homogeneity Level $80 \%$ (n5f80) (a) Initial Image (b) Ground Truth (c) Segmentation Result of FCM; (d) Segmentation Result Of Wells' Method (e) Segmentation Result of leemput's Method (f) Segmentation Result of li's Method (g)Segmentation Result of AFCM Method; (h) Segmentation Result Of Hidden-MR-F-Based Method; (i) Segmentation Result of NLFCM (j)

\section{Segmentation Result Of Our Method}

For comparison, we use Jaccard similarity (Js) as a metric to evaluate the performance of these methods. The Js is defined as the ratio between intersection and union of two sets and.

$$
J\left(S_{1}, S_{2}\right)=\frac{S_{1} \cap S_{2}}{S_{1} \cup S_{2}}
$$

where is the segmentation result and is the ground truth. A good algorithm should give high Js values. The average quantitative results of GM, WM and CSF when segmenting the whole simulated MRI data sets with different noise level are listed in Table.1.It is obvious that our method is more accurate than others.

Table 1. The Jaccard Similarity for Images

\begin{tabular}{|c|c|c|c|c|c|c|c|c|c|}
\hline & & FCM & Wells' & $\begin{array}{l}\text { Leemput } \\
\text { 's }\end{array}$ & Li's & AFCM & FSL & $\begin{array}{l}\text { NLFC } \\
\mathrm{M}\end{array}$ & ours \\
\hline \multirow{3}{*}{$\begin{array}{l}\quad \mathrm{N} 3 \\
\text { F80 }\end{array}$} & JS of WM & 84.58 & 90.32 & 89.11 & $\begin{array}{l}93.2 \\
6^{9} \\
\end{array}$ & 87.49 & $\begin{array}{l}93.2 \\
8^{9}\end{array}$ & 94.63 & 96.24 \\
\hline & JS of GM & 84.51 & 89.54 & 90.23 & $4^{92.5}$ & 83.92 & $\begin{array}{l}90.3 \\
0\end{array}$ & 93.51 & 94.87 \\
\hline & JS of CSF & 89.15 & 93.45 & 93.03 & $1^{94.2}$ & 85.57 & $5^{88.0}$ & 92.68 & 94.72 \\
\hline \multirow{3}{*}{$\begin{array}{l}\text { N5 } \\
\text { F80 }\end{array}$} & JS of WM & 80.25 & 77.70 & 81.66 & $\begin{array}{l}6^{85.7} \\
6\end{array}$ & 85.75 & $2^{88.0}$ & 93.41 & 94.13 \\
\hline & JS of GM & 78.93 & 76.01 & 83.61 & $5^{84.0}$ & 81.72 & $\begin{array}{l}{ }^{84.5} \\
1\end{array}$ & 91.26 & 91.97 \\
\hline & JS of CSF & 83.89 & 87.00 & 87.85 & $\begin{array}{l}8^{88.4} \\
\end{array}$ & 84.31 & $5^{86.2}$ & 88.97 & 92.12 \\
\hline \multirow{3}{*}{$\begin{array}{l}\text { N7 } \\
\text { F80 }\end{array}$} & JS of WM & 75.34 & 67.34 & 76.16 & $9^{76.0}$ & 84.20 & $\begin{array}{l}82.5 \\
5\end{array}$ & 89.23 & 90.94 \\
\hline & JS of GM & 72.35 & 65.89 & 76.81 & $2^{74.9}$ & 80.02 & $\begin{array}{l}0^{77.2} \\
0\end{array}$ & 86.18 & 88.72 \\
\hline & JS of CSF & 75.83 & 76.44 & 82.59 & $3^{80.3}$ & 83.96 & $\begin{array}{l}82.2 \\
3^{8}\end{array}$ & 86.46 & 87.42 \\
\hline
\end{tabular}

To compare with other methods, we utilize coefficient of variance (CV) as a metric to evaluate the performance of the algorithms for bias correction and segmentation. Coefficient of variance is defined as a quotient between standard deviation and mean value of selected tissue class. A good algorithm for bias correction and segmentation should give low CV values for the bias corrected intensities within each segmented region. We compared our method with the AFCM method, Wells' method, FSL method, SPM method [16], Leemput's method, Li's method and N3 method[10] on the data set N5F80.

The CV of GM and WM are shown in Table 2. It can be seen that the CV values of our 
method are lower than other methods, which indicates that the bias corrected images obtained in our method are more homogeneous than other methods. Figure4 shows the estimated bias fields of these eight methods. From these results we can find that our method can obtain more similar results.

Table 2. The Coefficient of Variance for Bias Corrected Images (N5F80)

\begin{tabular}{|c|c|c|c|c|c|c|c|c|c|}
\hline & N5F80 & $\begin{array}{c}\text { AF } \\
\text { CM }\end{array}$ & Wells' & FSL & SPM & $\begin{array}{c}\text { Leemput' } \\
\text { s }\end{array}$ & Li's & N3 & ours \\
\hline $\mathrm{M}$ & 0.1237 & $\begin{array}{c}0.0 \\
905\end{array}$ & 0.0882 & 0.0872 & 0.0861 & 0.0866 & 0.0859 & 0.0871 & $\begin{array}{c}0.085 \\
1\end{array}$ \\
\hline $\mathrm{M}$ & 0.1616 & $\begin{array}{c}0.1 \\
\mathrm{G}\end{array}$ & 0.1375 & 0.1372 & 0.1358 & 0.1368 & 0.1357 & 0.1369 & $\begin{array}{c}0.132 \\
4\end{array}$ \\
\hline
\end{tabular}

\subsection{Evaluation with Real Data}

In order to show the excellence of our method, we compared our method with other methods on a real 3T MR brain image data set, which is shown in Figure3.(a). The initial image has significant intensity in homogeneity and noise. Figure 3(b) shows the segmentation result of the FCM method. From the result we can find that due to the effect of the intensity in homogeneities, some pixels belonging to WM have be misclassified into GM and some pixels belonging to GM in the red regions have been misclassified into WM. The segmentation result of the Wells' method can be seen in Figure 3(c). In every iteration, the bias field should be smoothed by using low pass filter, which makes the Wells' method sensitive to strong bias field. Some pixels belonging to GM in the red circles have been misclassified into WM and some pixels belonging to CSF in blue region have been misclassified into GM. Compared to the Wells' method, the Leemput's method uses orthogonal polynomials to estimate the bias field and obtains more accurate result, which can be seen in Figure 3(d).However, the Leemput's only uses the intensity distribution information, which makes it sensitive to the noise. Figure 3(e) shows the segmentation result of the Li's method, which uses orthogonal polynomials as the basis function to estimate the bias field as well and obtain similar result. However, Li's method only utilizes the intensity distribution information, which makes it sensitive to the noise too. Due to the effect of the noise, pixels belonging to CSF in blue regions have been misclassified into GM. Furthermore, the Li's method is based on K-means method, which is less accurate than the Leemput's method.

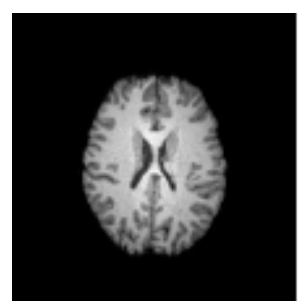

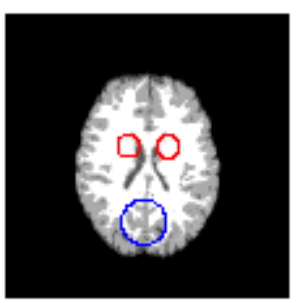

(b)

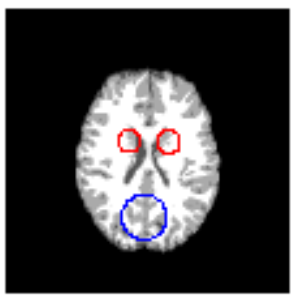

(c)

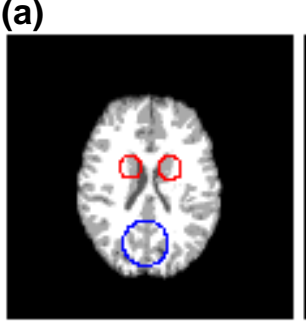

(d)

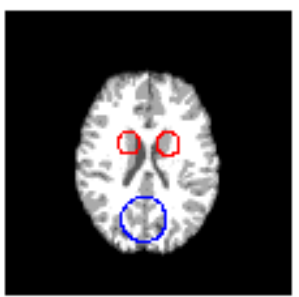

(e) 


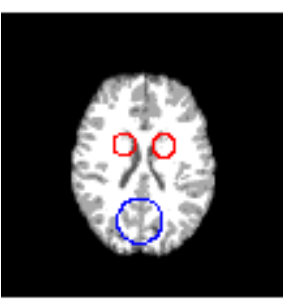

(f)

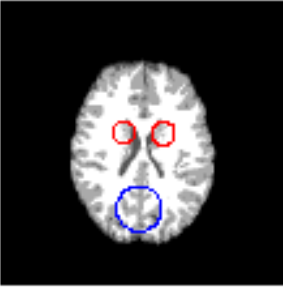

(g)

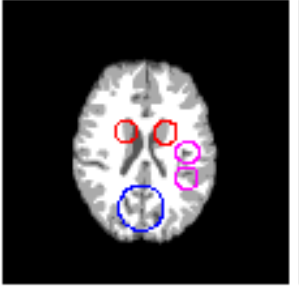

(h)

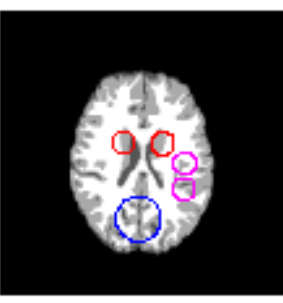

(i)

Figure 3. Segmentation Results On a 3t Brain MR Image (A) Initial Image (B) Segmentation Result Of FCM (C) Segmentation Result Of Wells Method (D) Segmentation Result Of Leemput's Method (E) Segmentation Result Of Li's Method (F)Segmentation Result Of AFCM Method (G) Segmentation Result Of Hidden-Mrf-Based Method (H)Segmentation Result Of NLFCM (I) Segmentation Result Of Our Method

Figure 3 (f-h) shows the segmentation results of the AFCM method, hidden-MRF-based method and NLFCM method, respectively. All these methods use region information to reduce the effect of the noise. However, the AFCM method uses low pass filter to smooth the bias field, which makes the method sensitive to the strong bias field and some pixels belonging to GM in red regions have been misclassified into WM. Further more, the AFCM method is a isotropic method which leads some pixels of the CSF in the blue region to be misclassified into GM. The hidden-MRF-based method can obtain more accurate result than that of the AFCM method. However, some pixels in the red and blue regions are still misclassified. It can be seen from the result of the NLFCM method that the method can more accurate result by using non-local information. However, this method needs to calculate local mean for each pixel in every iteration, which makes some pixels of the GM in the magenta region have been misclassified into CSF. Figure 3(i) shows the result of our method. From the result we can find that our method can obtain more accurate result than others.

In order to quantitatively evaluate the benefits, we segmented 20 standard sets of real brain MR data from the Internet Brain Segmentation Repository (IBSR at http://www.cma.mgh.harvard.edu/ibsr/ by using the FCM method, Wells' method, Leemput's method, Li's method, AFCM method, hidden-MRF-based method (FSL), NLFCM method and our method. The average quantitative results of GM, WM and CSF are listed in Table 3. It can be seen that our method is more accurate than others.

Table 3. The Jaccard Similarity For Real MR Images Segmentation

\begin{tabular}{|c|c|c|c|c|c|c|c|c|}
\hline & $\begin{array}{c}\mathrm{FC} \\
\mathrm{M}\end{array}$ & Wells' & Leemput's & Li's & $\begin{array}{c}\mathrm{AF} \\
\mathrm{CM}\end{array}$ & $\begin{array}{c}\begin{array}{c}\text { FS } \\
\mathrm{L}\end{array} \\
\end{array}$ & $\begin{array}{l}\text { NLF } \\
\mathrm{CM}\end{array}$ & ours \\
\hline JS of WM & $\begin{array}{l}37.0 \\
2\end{array}$ & 70.12 & 72.79 & $\begin{array}{c}73 . \\
17\end{array}$ & $\begin{array}{c}79 . \\
58\end{array}$ & $\begin{array}{c}82 . \\
34\end{array}$ & $\begin{array}{l}86.9 \\
2\end{array}$ & $\begin{array}{l}87.7 \\
6\end{array}$ \\
\hline JS of GM & $\begin{array}{l}49.7 \\
5\end{array}$ & 78.88 & 81.05 & $\begin{array}{c}79 . \\
18\end{array}$ & $\begin{array}{c}76 . \\
82\end{array}$ & $\begin{array}{c}82 . \\
19\end{array}$ & $\begin{array}{l}88.2 \\
4\end{array}$ & $\begin{array}{l}89.2 \\
1\end{array}$ \\
\hline JS of CSF & $\begin{array}{l}21.1 \\
8 \\
\end{array}$ & 65.5 & 68.82 & $\begin{array}{r}69 . \\
28 \\
\end{array}$ & $\begin{array}{c}57 . \\
12 \\
\end{array}$ & $\begin{array}{l}61 . \\
92 \\
\end{array}$ & \begin{tabular}{|l}
72.4 \\
5 \\
\end{tabular} & $\begin{array}{l}79.1 \\
1 \\
\end{array}$ \\
\hline
\end{tabular}

\subsection{Impact of the Non-Local Parameters}

In this paper, we use the non-local information to reduce the effect of the noise. should be updated by using non-local information in every iteration. However, the weight function will never change after it has been calculated, so it needs to be calculated only once. 


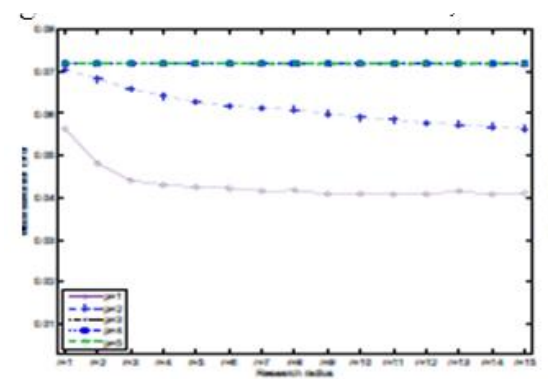

(a)

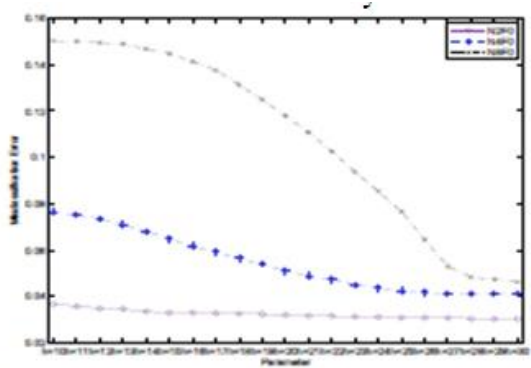

(b)

\section{Figure 4. Misclassified Error of the Segmentation with Different Parameters (a) Data N4F0 with Different $r$ and $P$ and $H=28$; (b) Data N2F0, N4F0 and N8F0 with Different $h$ and $p=1, r=6$.}

We analyzed the relationship between the parameters of Eq.4 and misclassification error (MCR), which is defined as the quotient between the number of pixels misclassified by the algorithm and the total number of pixels [26]. In this paper, we use image patches with $p=1$ and the radius of the search window is $r=6$. It is different with those in [38].The parameters will affect the accuracy of the segmentation results. Figure 4.(a) shows the MCRs with different parameters on a simulated image, whose noise level is $4 \%$ and in homogeneity level is zero. Figure4.(a) shows the MCR of the results with the different local region parameters and different search region parameters. We can see that when $p=1$ our method can obtain more precise results. It is because the brain tissues have more topological changes in the images. When $\mathrm{p}$ increases, it is hard to find some pixels with a similar neighborhood region. The weight function would be affected by the noise more easily. In [11], the author regarded that the research window sizes should be large enough to be robust to noise and meanwhile should be able to take care of the details and fine structure. However, from the experimental results we can find that when the radius is growing, the accuracy is not increasing; furthermore the increasing radius will augment the calculation time.

It is also possible to set the parameter automatically by using $h^{2}=2 \alpha \sigma^{2}\left|\Delta_{i}\right|$, which can be seen in [12]. In this formulation, the standard deviation of the noise $\sigma$, can be computed directly from the image. If the noise in the image is Gaussian, the parameter $\alpha$ can be fixed to 1; otherwise, it can be adjusted to provide a more accurate result. Figure4.(b) shows the influence of. We tested on three simulated images: N2F0, N4F0 and N8F0. It can be seen that the changes in have less effect on the accuracy when. Figure4.(b) shows the influence of $h$. We tested on three simulated images: N2F0, N4F0 and N8F0. In this experiment, we set $\mathrm{p}=1$ and $r=6$. It can be seen that the changes in $h$ have less effect on the accuracy when $h>27$.

\section{Conclusion}

In this paper, we have presented a new energy minimization framework for simultaneous estimation of the bias field and segmentation of tissues for magnetic resonance images. The algorithm was formulated by modifying the objective function of the standard FCM algorithm to compensate for intensity in homogeneities by using basis functions and to allow the labeling of a pixel (voxel) to be influenced by the nonlocal information. Results of simulated MRI data and real brain images show that intensity variations across patients, scans, and equipment changes have been accommodated in the estimated bias field without the manual intervention. Experimental results have shown that our method outperforms other segmentation methods when segmenting images with intensity in homogeneities and noise. 


\section{Acknowledgements}

This paper is a revised and expanded version of a paper entitled "A proved FCM for Brain MRI segmentation and bias estimation" presented at CST 2015, Suzhou, China, April 23-25, 2015. This work was supported by the Natural Science Foundation of Jiangsu Province (BY2014007-04), University Science Research Project of Jiangsu Province (13KJB520016), National Natural Science Foundation of China (614022342), and the Industrial Strategic Technology Development Program (10041740) funded by MOTIE Korea.

\section{References}

[1] J. B. Arnold, J. S. Liow, K. A. Schaper, J. J. Stern, J. G. Sled, D. W. Shattuck, A. J. Worth, M. S. Cohen R. M. Leahy, J. C. Mazziotta and D. A. Rottenberg, "Qualitative and quantitative evaluation of six algorithms for correcting intensity non uniformity effects", Neuro image, vol. 13, no. 3, (2011), pp. 931-943.

[2] U. Vovk, F. Pernus and B. Likar, "A review of methods for correction of intensity in homogeneity in MRI", IEEE Transactions on medical imaging, vol. 26, no. 3, (2007), pp. 405-421.

[3] G. Collewet, A. Davenel, C.Toussaint, and S. Akoka, "Correction of intensity nonuniformity in spin-echo t1weighted images", Magnetic resonance imaging, vol. 20, no. 4, (2002), pp. 365-373.

[4] W. Wells, W. Grimson and R. Kikinis, "Adaptive segmentation of mri data", IEEE Transactions on Image Process, vol. 15, no. 4, (1996), pp. 429-442.

[5] A. H. Andersen, Z. Zhang, M. J. Avison and D. M. Gash, "Automated segmentation of multispectral brain MR images", J. Neurosci Meth., vol. 12, no. 2, (2002), pp. 13-23.

[6] Y. Zhang, M. Brady and S. Smith, "Segmentation of brain mr images through a hidden markov random field model and the expectation-maximization algorithm", IEEE Transactions on Magn., vol. 20, no. 1, (2001), pp. 45-57.

[7] D. L. Pham and J. L. Prince, "An adaptive fuzzy c-means algorithm for image segmentation in the presence of intensity in homogeneities”, Pattern Recognition Letters, vol. 20, (1998), pp. 57-68.

[8] M. Ahmed, S. M. Yamany, N. Mohamed, A. Farag and T. Moriarty, "A modified fuzzy c-means algorithm for bias field estimation and segmentation of MRI data", IEEE Transactions on Medical Imaging, vol. 21, no. 2, (2002), pp. 193-199.

[9] K. Leemput, F. Maes and D. Vandermeulen, "Automated model-based bias field correction of MR images of the brain", IEEE Transactions on Image Process, vol. 18, no. 10, (1999), pp. 885-896.

[10] J. Sled, A. Zijdenbos and A. Evans, "A nonparametric method for automatic correction of intensity non uniformity in MRI data", IEEE Transactions on Image Process, vol. 17, no. 1, (1998), pp. 87-97.

[11] A. Buades, B. Coll and J. Morel, "A review of image denoising algorithms, with a new one", SIAM J. Multiscale Model. Simul., vol. 4, no. 2, (2005), pp. 490-530.

[12] P. Coupe, P. Yger, S. Hellier, C. Kervrann and C. Barillot, "An optimized block wise nonlocal means denoising filter for 3-d magnetic resonance images", IEEE Transactions on medical imaging, vol. 27, no. 4, (2008), pp. 425-440.

[13] J. Wang, J. -U. Kim, L. Shu, Y. Niu and S. Lee, "A distance-based energy aware routing algorithm for wireless sensor networks", Sensors, vol. 10, no. 10, (2010), pp. 9493-9511.

[14] J. Wang, Y. Yin, J. Zhang, S. Lee and R. S. Sherratt, "Mobility based energy efficient and multi-sink algorithms for consumer home networks", IEEE Transactions on Consumer Electronics, vol. 59, no. 1, (2013) February, pp.77-84.

[15] R. Hong, W. Cao, J. Pang and J. Jiang, "Directional projection based image fusion quality metric. Inf. Sci.", (2014), pp. 611-619.

[16] R. Hong, L. Tang, J. Hu, G. Li and J. Jiang, “Advertising object in web videos. Neurocomputing, (2014), pp.118-124.

[17] C. Li, C. Gatenby, L. Wang and J. C. Gore, "A robust parametric method for bias field estimation and segmentation of MR images", in CVPR, (2009), pp. 218 - 223.

[18] C. Li, R. Huang, Z. Ding, G. C, D. Metaxas and J. Gore, "A variation level set approach to segmentation and bias correction of images with intensity in homogeneity", in MICCAI, (2008), pp. 1083-1091.

[19] B. Caldairou, F. Rousseau, N. Passat, P. Habas, C. Studholme and C. Heinrich, "A non-local fuzzy segmentation method, Application to brain MRI", in CAIP'09, (2009), pp. 606-613.

[20] J. Ashburner, "Another mri bias correction approach", in 8th International Conference on Functional Mapping of the Human Brain, vol. 16, (2002).

[21] Y. Chen and Z. Wang, "An proved FCM for Brain MRI segmentation and bias estimation", Proceedings of international conferences on ISA, CIA 2014, Philippines, vol. 48, (2014), pp. 95-102. 


\section{Authors}

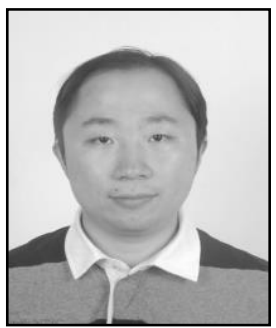

Yunjie Chen, received his PHD degree in 2008 from Nanjing University of Science \& Technology. His main research interests include image processing, pattern recognition and numerical analysis.

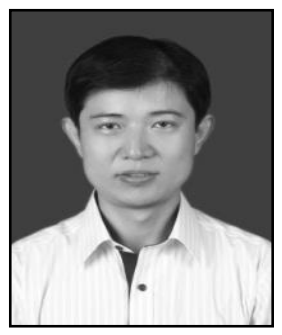

Jin Wang, received the B.S. and M.S. degree from Nanjing University of Posts and Telecommunications, China in 2002 and 2005, respectively. He received Ph.D. degree from Kyung Hee University Korea in 2010. Now, he is a professor in the Computer and Software Institute, Nanjing University of Information Science and technology. His research interests mainly include routing method and algorithm design, performance evaluation and optimization for wireless ad hoc and sensor networks. He is a member of the IEEE and ACM.

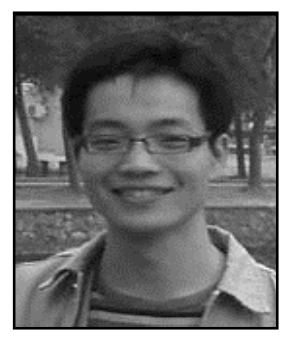

Yuhui Zheng, received his PHD degree in 2009 from Nanjing University of Science \& Technology. His main research interests include image processing, pattern recognition and numerical analysis. 
International Journal of Grid Distribution Computing Vol. 8, No.4, (2015) 